\title{
Enhanced optical transmission through tapered metallic gratings
}

Honghui Shen ${ }^{1}$ and Bjorn Maes ${ }^{1,2}$

1) Photonics Research Group (INTEC), Ghent University-IMEC, Sint-Pietersnieuwstraat 41, 9000 Gent, Belgium ${ }^{\mathrm{a})}$

2) Micro- and Nanophotonic Materials Group, Faculty of Science, University of Mons,

Bat. IV, Avenue Maistriau 19, B-7000 Mons, Belgium

(Dated: 25 May 2012)

To achieve non-resonant behavior, we propose a metallic grating device with linearly tapered slits. The tapering provides a gradual impedance variation from the entrance to the exit of the slits, leading to broadband and wide-angle enhanced transmission in the infrared. In addition, the light is strongly localized and enhanced at the slit exits, in contrast with straight slits. We describe the phenomenon with a transmission line model, that is in accordance with rigorous simulations.

Keywords: tapered slit, enhanced transmission, transmission line model

a)Electronic mail: honghui.shen@intec.ugent.be. 
The extraordinary optical transmission (EOT) phenomenon was observed in an opaque metallic film with a periodic array of subwavelength holes in $1998^{1}$. Since then tremendous investigation has been dedicated to its mechanism ${ }^{2-5}$, elucidating the role of surface plasmon dispersion ${ }^{6}$ and Fabry-Perot type cavity modes $^{7}$. EOT offers strongly localized field enhancements in addition to a large transmission, with potential applications in nonlinear optics, sensing etc. However, due to the resonant mechanisms involved often a relatively narrow bandwidth is affected, which is unsuitable for a range of applications. On the other hand, broadband transparency has been achieved in related structures, e.g. using oblique incidence TM polarization ${ }^{5,8}$, or by connecting rectangular apertures with narrower slits ${ }^{9}$. In the latter example the thin gratings operate at wavelengths beyond the Fabry-Perot resonances of guided modes in the slits. This regime of non-resonant devices has only recently attracted attention, but it is indispensable e.g. for broadband light harvesting devices.

Therefore, we propose metallic gratings with tapered slits, which offer a much broader transmission window for TM polarized light. By gradually varying the impedance from input to output plane, we effectively destroy the Fabry-Perot type resonant conditions of guided modes in the slit, yielding a non-resonant and thus broadband and wide-angle large transmission in the infrared. In addition, the localization of the field is confined to one plane of the structure, instead of over the whole lossy waveguide as in the traditional structures with straight sidewalls.

Analysis is performed with rigorous calculations (finite element method - FEM ${ }^{10}$ ) and semi-analytic methods (transmission line theory - $\mathrm{TL}^{8}$ ). Other more complex models and theories were proposed for metallic aperture devices ${ }^{11,12}$, but under certain conditions the efficient TL model provides effective characterization. To illustrate the effects of tapering we assume surrounding, substrate and slit material to be air. The standard geometry and the tapered grating are shown in Fig. 1(a) and (b), respectively. The grating with tapered slits is characterized by the thickness $t$, period $p$, and widths $w_{\text {in }}$ and $w_{\text {out }}$ at the entrance and exit of the slit, respectively. The metal grating is assumed to be silver ${ }^{15}$. Fig. 2 shows the calculated TM transmission $T$ with normal incidence $(\theta=0)$ for grating structures with different sizes, with both FEM and TL calculations. For various parameters, the tapered grating leads to a dramatic enhancement of the transmission over a broadband wavelength range, comparing the straight sidewall case (Fig. 2, blue curves) with the tapered cases. In addition, we notice that the tapered transmission seems to average the Fabry-Perot resonant 
peaks for the non-tapered geometry, leading to non-resonant transmission. This averaging effect is clear for the smaller wavelengths, and becomes more pronounced for larger $w_{\text {in }}$ (more 'open' gratings).

We introduce now the employed TL approach, which was developed for non-tapered slits ${ }^{8}$. Surrounding and substrate are modeled as semi-infinite TLs characterized by a wavenumber $\left(\beta_{i n}, \beta_{s}\right.$, respectively) and a characteristic impedance per unit length $\left(Z_{\text {in }}, Z_{s}\right.$, respectively), see circuit in Fig. 1(a). The wavenumber is given by $\beta_{u}=k_{0} n_{u} \cos \theta$, the characteristic impedance is $Z_{u}=Z_{0} p \cos \theta / n_{u}, u=i n$ (surrounding), $s$ (substrate), and $Z_{0}=\sqrt{\mu_{0} / \epsilon_{0}}$ is the vacuum impedance. The grating layer is treated as a TL characterized by $\beta$ and $Z$ with finite length $t$. Given that the metal width $d(=p-w)$ is large enough to avoid coupling between slits, the wavenumber $\beta$ is determined by the dispersion relation of a metal-insulator-metal waveguide ${ }^{13}$ :

$$
\tanh \left(\sqrt{\beta^{2}-k_{0}^{2} n_{2}^{2}} w / 2\right)=-\frac{\sqrt{\beta^{2}-k_{0}^{2} n_{1}^{2}} n_{2}^{2}}{\sqrt{\beta^{2}-k_{0}^{2} n_{2}^{2}} n_{1}^{2}}
$$

Then the characteristic impedance per unit length can be expressed $\operatorname{as}^{8}$

$$
Z=w \beta /\left(\omega \epsilon_{0}\right)
$$

The tapered grating is modeled as a series of cascaded TLs, with a staircase multilayer characterized by local widths $w_{i}$ and thicknesses $t_{i}$ (circuit in Fig. 1(b)). To calculate the transmission $T$ of the cascaded network one can use $A B C D$ parameters (also known as transfer matrix $)^{14}$. The $A B C D$ parameters $(M)$ of the whole network are a multiplication of each TL in the network: $M=\left[A_{t o t} B_{t o t} ; C_{t o t} D_{t o t}\right]=\prod M_{i}=\prod\left[A_{i} B_{i} ; C_{i} D_{i}\right]$, where $A_{i}=D_{i}=\cos \left(\beta_{i} t_{i}\right), B_{i}=j Z_{i} \sin \left(\beta_{i} t_{i}\right), C_{i}=j Y_{i} \sin \left(\beta_{i} t_{i}\right), Y_{i}=1 / Z_{i}, i=1,2, \cdots, N$, $(i=1,2, \cdots, N)$. Therefore $T$ is expressed by

$$
T=\frac{4\left|Y_{i n}\right|^{2} \operatorname{Re}\left(Y_{s}\right)}{\left|m_{1} Y_{i n}+m_{2}\right|^{2} \operatorname{Re}\left(Y_{i n}\right)}
$$

where $m_{1}=A_{t o t}+B_{t o t} Y_{s}, m_{2}=C_{t o t}+D_{t o t} Y_{s}, Y_{u}=1 / Z_{u}, u=i n, s$. Upon derivation of Eq. 2 uniform electric fields in the slit and negligible coupling between the slits are assumed ${ }^{8}$, so the TL model works well with small slit widths $(w)$ and large enough metal widths $(d)$. In addition, diffraction in the surrounding and substrate, and surface modes at the grating planes are not considered in the TL model, so the period of the grating cannot be larger than the wavelength. 
The plots in Fig. 2 show a good agreement between the rigorous full-wave FEM simulations and the TL model. Here, in the TL model 10 layers are used and already provide a good convergence. More in detail, agreement is better for small periods (Fig. 2(a)), and for straight slits (blue curves in Fig. 2(a) and (b)). The deviation is mainly caused by plasmon modes at the slit corners, which are not taken into account in the TL model. Deviation increases when $w_{\text {in }}$ increases, since the coupling between slits becomes stronger, and uniformity of the $E$ field in the slits diminishes. However, the TL model still predicts the trend of $T$ very well, except for an overestimation at smaller wavelengths.

Based on the large transmission enhancement by tapering we can imagine that the light is slowly squeezed from the entrance of the taper on to the narrower exit slit. Therefore the field is expected to be gradually enhanced. To confirm we plot the electric field spatial distribution (Fig. 3) at wavelength $5 \mu \mathrm{m}$ for gratings corresponding to Fig. 2(b). For other grating sizes the field spatial distribution profiles are very similar. We notice indeed that the $E$ amplitude gradually increases towards the exit of the slit by tapering, contrast (a) to (b) and (c). In addition, the fields reach their maximum exactly at the exit of the tapered slit, and the maximum value is much larger than in the straight sidewall case.

To examine the field enhancement properties Fig. 4 shows the average normalized electric field as a function of wavelength at the slit exit (same gratings as in Fig. 3). Broadband field enhancement is obtained, and it increases as the taper becomes wider (increasing $w_{i n}$ ). Meanwhile the spectrum of the normalized field is similar to the corresponding transmission spectrum (Fig. 2(b)). Therefore the near-field at the exit of the slit and the transmission have a similar spectrum, with deviations from evanescent wave components. In addition, for larger wavelengths we notice that the normalized electric field arrives quite close to the dash-dotted line, which is the ratio of $p / w_{\text {out }}$, similar to Ref. [9]. The average field decreases towards smaller wavelengths, since there is less light transmitted. However, by tapering it is still possible to obtain a local field enhancement far beyond the ratio of $p / w_{\text {out }}$, instigated by the sharp corner at the exit of the slit, as the field maxima show in Fig. 3. Therefore tapering offers a strong control over the field enhancement profile, by tailoring the transmission spectrum, the value of $p / w_{\text {out }}$ and the corner sharpness.

Finally, we compare the angular response of the straight sidewall gratings (Fig. 5(a)) with the tapered gratings (Fig. 5(b)). For the straight sidewall (Fig. 5(a)) we observe that the transmission gradually increases with the angle, and reaches its maximum near the dashed 
line around $80^{\circ}$, which is the brewster angle $\theta_{B}$ determined by impedance matching between the surrounding and the straight slit $^{8}$. For the tapered case (Fig. 5(b)) we see that the overall transmission is significantly larger. In addition, we still observe brewster angle behavior, i.e. the transmission increases with incident angle, although the impedance in the slit is not uniform.

In experimental realizations a different substrate may be necessary. Our calculations show the validity of the tapering idea for $n_{s} \neq n_{i}$, e.g. with a glass substrate, except that the transmission at larger wavelength is determined by that of light through a semi-infinite surrounding and substrate $\left(T=4 n_{s} n_{i n} /\left(n_{s}+n_{i n}\right)^{2}\right)$.

In summary, we demonstrate the concept of tapering in metallic gratings to achieve broadband and wide-angle transmission. In addition, the taper provides a strong enhancement and localization of light at the exit of the slit, useful for applications such as nonlinear optics, light harvesting, sensing ${ }^{16}$ and emission enhancement. The TL model gives a very efficient characterization of the tapering effect, therefore it can be used to assist more complex designs, e.g. with parabolic shapes. Finally, the transmission at smaller wavelengths is further improved by tilting the incident light, giving rise to a plasmonic brewster type effect. This type of structure therefore expands the field of non-resonant gratings and broadband transmission.

The authors thank Y. Li for useful discussions. This research was supported by the IWT (Institute for the Promotion of Innovation by Science and Technology in Flanders) via the SBO-project No. 060843 'Polyspec', by the Interuniversity Attraction Poles program of the Belgian Science Policy Office under Grant No. IAP P6-10 'photonics@be' and by COST MP 0702 .

\section{REFERENCES}

${ }^{1}$ T. W. Ebbesen, H. J. Lezec, H. F. Ghaemi, T. Thio, and P. A. Wolff, Nature 391, 667 (1998).

${ }^{2}$ H. Ghaemi, T. Thio, D. Grupp, T. Ebbesen, and H. Lezec, Phys. Rev. B 58, 6779 (1998).

${ }^{3}$ J. Porto, F. García-Vidal, and J. Pendry, Phys. Rev. Let. 83, 2845 (1999).

${ }^{4}$ L. Martín-Moreno, F. García-Vidal, H. Lezec, K. Pellerin, T. Thio, J. Pendry, and T. Ebbesen, Phys. Rev. Let. 86, 1114 (2001). 
${ }^{5}$ X.-R. Huang, R.-W. Peng, and R.-H. Fan, Phys. Rev. Let. 105, 243901 (2010).

${ }^{6}$ W. L. Barnes, A. Dereux, and T. W. Ebbesen, Nature 424, 824 (2003).

${ }^{7}$ Q. Cao and P. Lalanne, Phys. Rev. Let. 88, 057403 (2002).

${ }^{8}$ A. Alù, G. D'Aguanno, N. Mattiucci, and M. Bloemer, Phys. Rev. Let. 106, 123902 (2011).

${ }^{9}$ G. Subramania, S. Foteinopoulou, and I. Brener, Phys. Rev. Let. 107, 163902 (2011).

${ }^{10}$ Comsol Multiphysics.

${ }^{11}$ F. Medina, F. Mesa, and R. Marqués, IEEE Trans. Microwave Theory Tech. 56, 3108 (2008).

${ }^{12}$ V. Delgado and R. Marqu, Opt. Express 19, 25290 (2011).

${ }^{13}$ E. N. Economou, Phys. Rev. 182, 539 (1969).

${ }^{14}$ D. M. Pozar, Microwave Engineering (Wiley, Danvers, 2005).

${ }^{15}$ E. D. Palik, Handbook of Optical Constants of Solids (Academic, New York, 1985).

${ }^{16}$ M. Schnell, P. Alonso-González, L. Arzubiaga, F. Casanova, L. E. Hueso, A. Chuvilin, and R. Hillenbrand, Nat. Photonics 5, 283 (2011). 

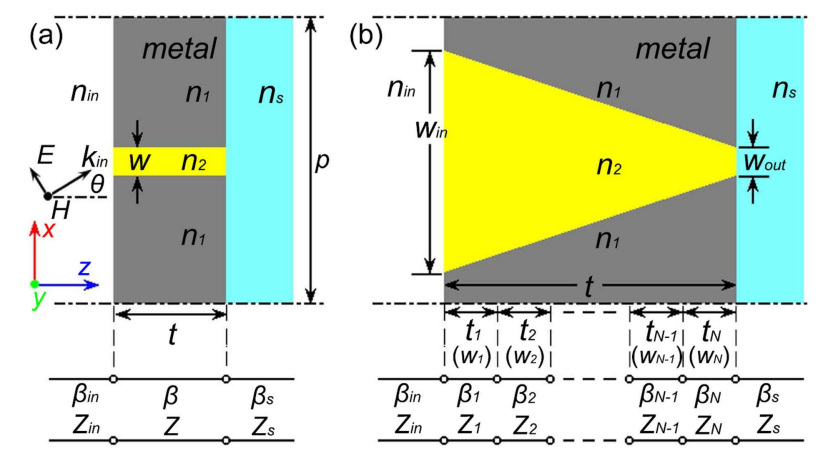

FIG. 1. (Color online) Schematics of 1D metallic grating with (a) straight slits, and (b) linearly tapered slits, respectively. Together with illustrations of the TL model underneath. The gratings are periodic in the $x$-direction.

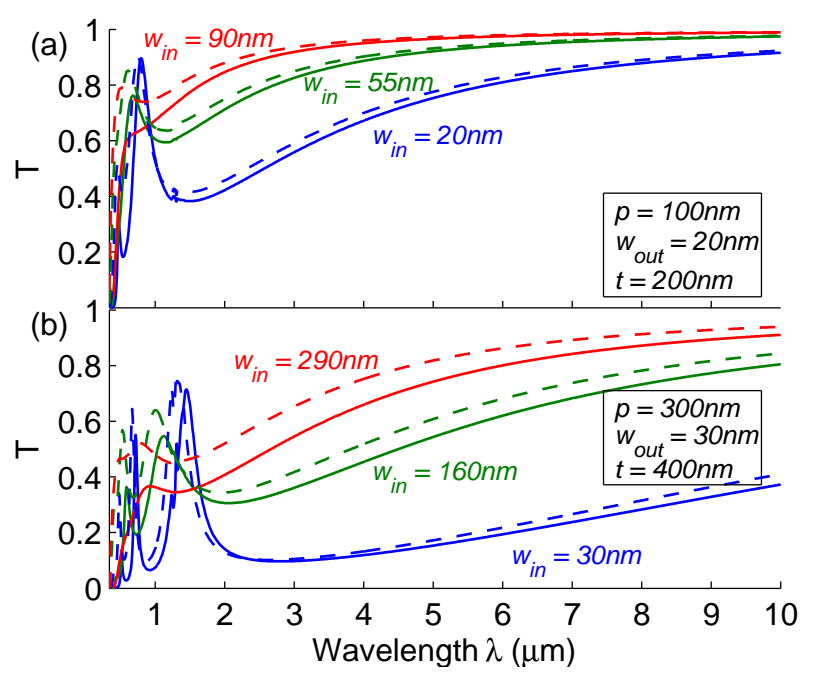

FIG. 2. (Color online) Transmission $\mathrm{T}$ for gratings with different sizes (i.e., different slit width $w_{\text {in }}$ and $w_{\text {out }}$, grating period $p$, slit thickness $t$ ). The solid lines are from numerical simulation by FEM. The dash lines are from TL model. 

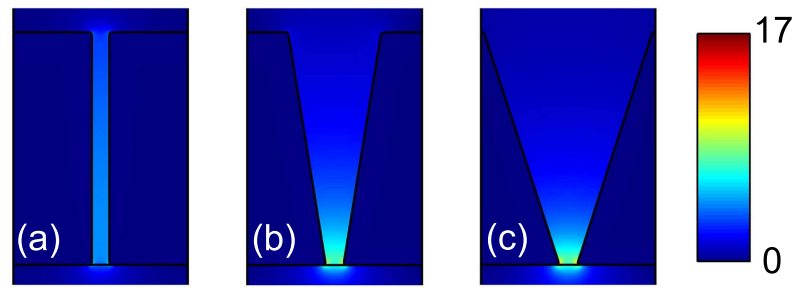

FIG. 3. (Color online) E field amplitude (normalized by the incident field amplitude $E_{0}$ ) distribution at $\lambda=5 \mu \mathrm{m}$ for gratings $\left(t=400 \mathrm{~nm}, w_{\text {out }}=30 \mathrm{~nm}, p=300 \mathrm{~nm}\right)$ with (a) $w_{\text {in }}=30 \mathrm{~nm}$, (b) $w_{\text {in }}=160 \mathrm{~nm},(\mathrm{c}) w_{\text {in }}=290 \mathrm{~nm}$.

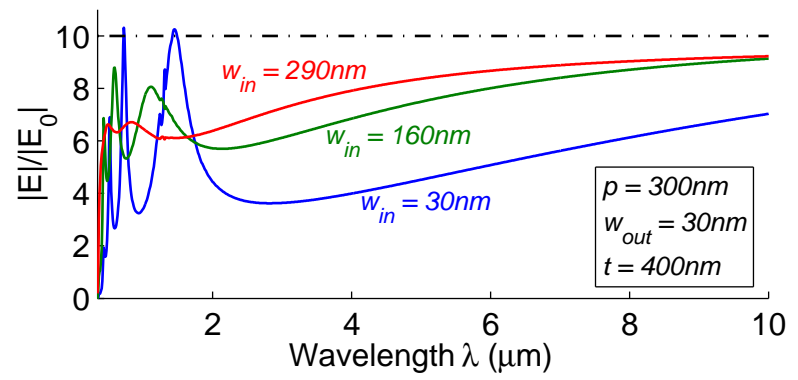

FIG. 4. (Color online) Average E field amplitude (normalized by the incident field amplitude $E_{0}$ ) versus wavelength at the exit of slit for gratings $\left(t=400 \mathrm{~nm}, w_{\text {out }}=30 \mathrm{~nm}, p=300 \mathrm{~nm}\right)$ with different widths: $w_{i n}=30 \mathrm{~nm}$ (blue), $w_{i n}=160 \mathrm{~nm}$ (green), and $w_{i n}=190 \mathrm{~nm}(\mathrm{red})$.

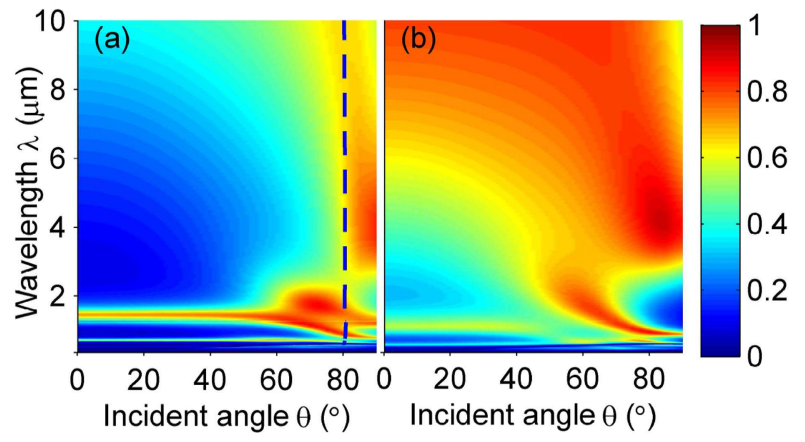

FIG. 5. (Color online) Angular response for gratings $\left(t=400 \mathrm{~nm}, p=300 \mathrm{~nm}, w_{\text {out }}=30 \mathrm{~nm}\right)$ with (a) $w_{\text {in }}=30 \mathrm{~nm}$ (straight sidewalls), (b) $w_{\text {in }}=160 \mathrm{~nm}$ (tapered sidewalls). The dashed line in (a) is the plasmonic brewster angle. 